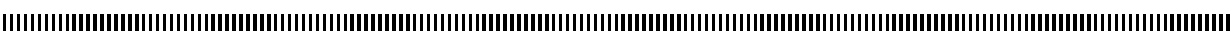

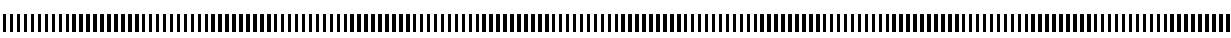
|

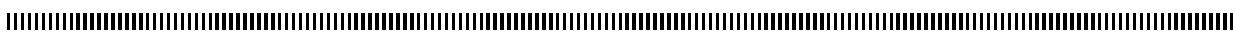
|

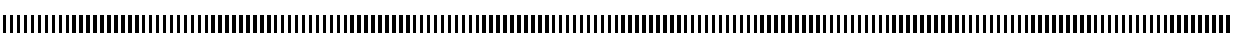
|

\title{
Canard solutions and bifurcations in smooth models of plane structure variable systems
}

\author{
Luis Gonzaga Albuquerque
}

Universidade Aberta

Rua da Escola Politécnica 147

1269-001 Lisboa, Portugal

lgalbu@univ-ab.pt

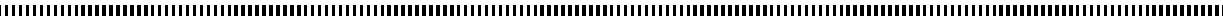

ABSTRACT. Systems that operate in different modes with quick transition are usually studied through discontinuous systems. We give a model of a smoothing of the transition between two vector fields along a separation line, allowing perturbations of the vector fields and of the separation line. In this model there appears a canard phenomenon in certain macroscopically indeterminate situations. This phenomenon gives a new point of view on some situations usually studied through discontinuous bifurcations. We also study the dynamics near the transition line through an associated slow-fast system and compare the slow dynamics with the classical theory, namely, sliding mode dynamics in variable structure systems and equivalent control.

RÉSUMÉ. Le raccordement des trajectoires de deux champs de vecteurs donnés de part et d'autre d'une courbe dans le plan, est fait d'habitude à travers des champs discontinues. On donne un modèle de lissage de ce raccordement, qui fait apparaître des phénomènes canard, dans certaines situations macroscopiquement indeterminés. On utilise un champ lent-rapide associé au modèle de raccordement, pour étudier les dynamiques dans un voisinage infinitésimal de la ligne de transition et on fait un étude comparative avec la théorie classique, notamment: mouvements glissants dans les systèmes de structure variable et contrôle équivalent.
\end{abstract}

KEYWORDS : Canards, slow-fast systems, nonstandard analysis, sliding mode dynamics, equivalent control, discontinuous bifurcations

MOTS-CLÉS : Canards, systèmes lent-rapides, analyse non standard, mouvement glissant, contrôle équivalent, bifurcations discontinues.

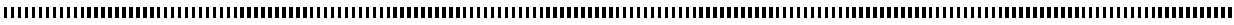




\section{Introduction}

Many systems operate in different modes with nearly instantaneous transition from one mode to another. Such systems (dry friction, electronic control systems, economic dynamics...) have been studied since the thirties $[2,10,11]$. The transitions are usually modelled by discontinuous systems, where the trajectories of two plane vector fields $X_{1}$ and $X_{2}$ are connected continuously across some transition line $C$, whenever the topology of the trajectories on both sides of $C$ determines the connections uniquely. Concerning the uniqueness of the solutions, the problem remains open in other cases, e.g. that of FIG. 1 , where there is no topological criterion to extend a trajectory of $X_{1}$ or $X_{2}$, starting on the left, beyond the point $P$.

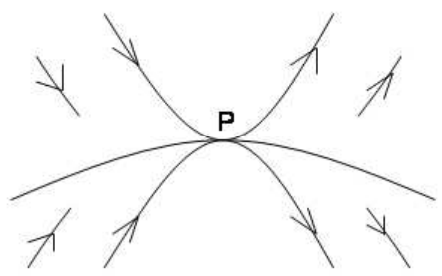

Figure 1. Indeterminate situation: there is no criterion to extend a trajectory, starting on the left, beyond the point $P$.

In this problem there is no uniqueness of solutions within classical Filippov's approach, indeed one solution starting on the left after arriving at the point $P$, may escape either towards the top or towards the bottom, or remain on the transition line and escape towards the top or the bottom at any moment [9]. In this paper we approach the problem by smoothing the transition between $X_{1}$ and $X_{2}$ across the line $C$ and compare our model with Filippov's theory. Roughly speaking we obtain a field $Z$, named collage of fields such that, outside a narrow band around the separation line, $Z$ is almost $X_{1}$ or $X_{2}$, and study the dynamics near the transition line through an associated slow-fast system. We allow perturbations of the vector fields $X_{i}$ and of the curve $C$.

We work within Nelson's Internal Set Theory [15] and we use systematically the results on canard phenomena obtained by E. Benoît, J.L. Callot, M. and F. Diener [3, 4, 6, $7,8,13,14,20]$, due to the fact that in situations like that in FIG. 1 canard trajectories can appear in the associated slow-fast system.

We consider slow-fast systems of the form

$$
\left\{\begin{array}{l}
\epsilon \dot{x}=f(x, y, a) \\
\dot{y}=g(x, y, a)
\end{array}\right.
$$

with $\epsilon$ small and positive, $f, g$ being smooth and standard functions. The behavior of its solutions is determined by the slow curve $C$ defined by $f\left(x, y,{ }^{0} a\right)=0$, with ${ }^{0} a$ the unique standard real number infinitely close to $a$. 
Indeed outside a narrow vicinity of $C, x$ varies fast and the solution approaches almost horizontally an attractive point $\left(x_{0}, y_{0}\right)$ of the slow curve [a point where $f_{x}\left(x_{0}, y_{0},{ }^{0} a\right)<$ $0]$ and it remains in a small vicinity of $C$ until it reaches a critical point $\left[f_{x}\left(x_{0}, y_{0},{ }^{0} a\right)=\right.$ $0]$, where usually it "jumps" to another attractive point.

Exceptionally, canard solutions can arise, i.e. solutions that follow two consecutive arcs of the slow curve, the first being attractive and the second repulsive [a point of the slow curve is repulsive if $f_{x}\left(x_{0}, y_{0},{ }^{0} a\right)>0$ ]

This is an unexpected and unstable phenomenon, which was first discovered with the study of the limit cycle amplitude evolution in the forced Van der Pol's equation,

$$
\epsilon \ddot{x}+\left(x^{2}-1\right) \dot{x}+x=a
$$

with respect to the value of the parameter $a, \epsilon$ being infinitesimal. Duck-shaped curves appeared in the Lienard's phase plane of this last equation, which is the slow-fast system

$$
\left\{\begin{array}{l}
\epsilon \dot{x}=y-\frac{x^{3}}{3}+x \\
\dot{y}=a-x
\end{array}\right.
$$

obtained through the change of variable $y=\epsilon \dot{x}+\frac{x^{3}}{3}-x$.

A dramatic change in the amplitude and period of the limit cycles occurs for values of $a$ near 1 , indeed for $a<1$ and $a$ not near 1 , all limit cycles being approximately the same (see FIG. 2, left) disappearing abruptly for $a=1$ where a Hopf -Andronov bifurcation occurs. Similar problems have been studied by Takens from a discontinuous point of view, through the use of constrained equations [7, 18, 19]. The nonstandard approach through the existence of canards gave a continuous description of the system's behavior during the change between the great cycles and infinitesimal cycles before the occurrence of the Hopf-Andronov bifurcation (see FIG. 2, right).
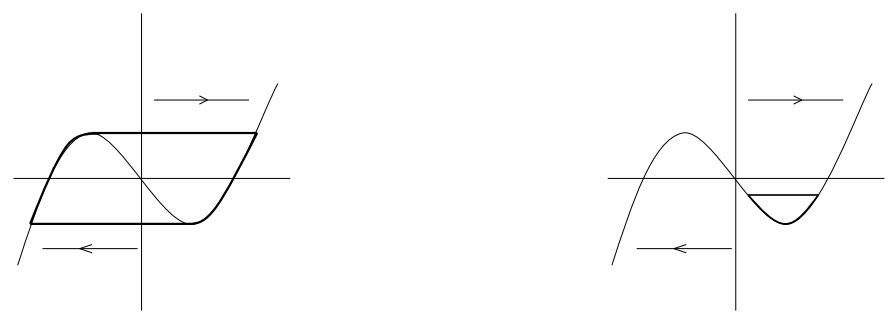

Figure 2. Limit cycle of the van der Pol's equation. On the left, the case $a<1$ and a not near 1 . On the right a canard limit cycle that follows two consecutive arcs of the slow curve, the first one being attractive and the second one repulsive.

Similarly in situations like that described in FIG. 1 where we allow perturbations of the vector fields and of the transition line, the existence of canards in an associated slowfast system gives a continuous description of the system's behavior in contrast with the classical discontinuous bifurcations approach [12].

As a by-product, we prove that when the transition line in collage of fields is attractive, the slow dynamics of the associated slow-fast system is infinitely close to the sliding 
motion in Fillipov's theory, when all data are standard. When at least one of the fields is nonstandard, slow dynamics and sliding motion can be different.

The use of discontinuous control in control theory gives rise in a natural way to variable structure systems but, as Utkin remarks, Fillipov's solutions do not take account of all types of motions produced under discontinuous control. Following the same philosophy used in collage of fields we give a model that smooths discontinuous control and we obtain equivalent control dynamics as slow motions of an associated slow-fast system.

This article is organized as follows: in Section 2 we give basic assumptions on collage of fields, necessary conditions on the geometrical configuration, which allow the presence of canard trajectories and we end with a significant example where the existence of canards is exemplified in detail. In Section 3 we study generic situations where canards are present. Section 4 is devoted to a comparative study between slow dynamics obtained in collage of fields and sliding motion in Fillipov's theory and Utkin's equivalent control. In Section 5 we study bifurcations on collage of fields and make a comparative study with discontinuous bifurcations approach as formulated in $[9,12]$ namely through the canard phenomenon.

\section{Collage of fields}

\subsection{Basic assumptions}

In this section we begin with basic assumptions on collage of fields. Roughly speaking we give a class of functions that smooths the discontinuity in the sign function and we use it to define collage of fields.

Nonstandard Analysis has a privileged place in this process once the difference between continuity and S continuity allows the existence of smooth models of quick changing situations, where usually a discontinuous approach is used.

More precisely we introduce a standard $C^{\infty}$ function $\varphi$ from the real line on to ]-1, 1[ such that $\varphi(0)=0, \varphi^{\prime}(0)=1, \varphi^{\prime}(x)>0$ for all $x$ and $\varphi(x) \rightarrow \pm 1$ when $x \rightarrow \pm \infty$.

When $\omega$ is a fixed unlimited positive integer, the $C^{\infty}$ function $\varphi(\omega x)$ is a smooth model for the signum function. Indeed if $x \gg 0$ then $\varphi(\omega x) \simeq 1$ and if $x \ll 0$ then $\varphi(\omega x) \simeq-1$, (write $\simeq$ for the infinitesimal proximity, $a \gg b$ for $a>b$ and not $a \simeq b$ ). In between, the function jumps in a smooth way. Clearly $\varphi(\omega x)$ is a $C^{\infty}$ function but not $\mathrm{S}$ continuous.

Let $X_{i=1,2}^{a}$ be two standard $C^{\infty}$ one-parameter families of vector fields defined by

$$
X_{i}^{a}\left\{\begin{array}{l}
\dot{x}=f^{i}(x, y, a) \\
\dot{y}=g^{i}(x, y, a)
\end{array}\right.
$$

with $f^{i}, g^{i}$ standard functions and let $C_{a}$ be a one-parameter family of transition curves defined by $h(x, y, a)=0$, where $h$ is a standard $C^{\infty}$ function. Suppose that, for each $a, 0$ is a regular value of $h(x, y, a)=0$ and that $C_{a}$ divides the plane in two open connected components such that $h(x, y, a)>0$ on $D_{1}^{a}$ and $h(x, y, a)<0$ on $D_{2}^{a}$.

In what follows we suppose that $a$ is near standard with shadow ${ }^{0} a$, and $\omega$ is a fixed unlimited positive integer. 
Definition 2.1. The vector field

$$
Z^{a}=\frac{1+\varphi(\omega h(x, y, a))}{2} X_{1}^{a}(x, y)+\frac{1-\varphi(\omega h(x, y, a))}{2} X_{2}^{a}(x, y)
$$

is called the collage of the fields $X_{1}^{a}, X_{2}^{a}$ through the transition line $C_{a}$. The associated slow-fast system is the system obtained through the change of variable $v=\omega h(x, y, a)$.

Under these conditions, when $h(x, y, a) \gg 0$ we have $Z^{a} \simeq X_{1}^{a}$ and when $h(x, y, a) \ll$ 0 we have $Z^{a} \simeq X_{2}^{a}$. In between there is a smooth transition.

Limited points of the plane $(x, v)$ correspond to points of the $(x, y)$ plane that belong to the $\frac{1}{\omega}$ galaxy $\left(C_{a}\right)=\{(x, y, a): \omega h(x, y, a)=L\}$ where $L$ sands for a limited number. So the study of the dynamics of the collage of fields in the $\frac{1}{\omega}$ galaxy $\left(C_{a}\right)$ can be done through the associated slow-fast system.

Definition 2.2. We say that a trajectory of $Z^{a}$ follows a standard arc I of $C_{0}$ if its shadow is $I$. Two disjoint arcs $I$ and $J$ with at least one common accumulation point are called consecutive.

In the sequel we suppose $I$ and $J$ standard, and $\langle u, v\rangle$ stands for the scalar product of the vectors $u$ and $v$.

Definition 2.3. Let $I^{a}$ be an arc of the curve $C_{a}$. The field $X_{1}^{a}$ is called outgoing from $D_{1}^{a}$ along $I^{a}$ whenever

$$
\left\langle X_{1}^{0} a(x, y), \operatorname{grad}\left(h\left(x, y,{ }^{0} a\right)\right)\right\rangle<0
$$

on I where I is the shadow of $I^{a}$; it is called incoming to $D_{1}^{a}$ whenever

$$
\left\langle X_{1}^{0} a(x, y), \operatorname{grad}\left(h\left(x, y,{ }^{0} a\right)\right)\right\rangle>0
$$

on I. Similarly for $X_{2}^{a}$ and $D_{2}^{a}$.

Definition 2.4. If both fields $X_{1}^{a}, X_{2}^{a}$ are outgoing (resp. incoming) along an arc $I^{a}$, this arc is called attractive (resp. repulsive). A trajectory of the field $Z^{a}$ is called a canard trajectory whenever it follows two consecutive arcs I and $J$ of $C_{0}{ }_{a}$, the first one attractive the second one repulsive, and a faux canard whenever the first one is repulsive and the second one attractive.

\subsection{Macroscopic necessary condition}

In order to get necessary conditions for the existence of canards, we use the following

Lemma 2.5. Let $X=\left(\begin{array}{l}A(x, y) \\ B(x, y)\end{array}\right)$ be a standard $C^{\infty}$ planar vector field, with $A(0,0)>0$ and $B(0,0)=0$. Let $y=\gamma(x)$ be the orbit of $X$ containing $(0,0)$ and let $y=f(x)$ be a standard $C^{\infty}$ function such that $f(0)=f^{\prime}(0)=0$. Suppose that at $(0,0)$ both functions have a contact of finite order $n$. Then in some neighborhood of the origin and for $h(x, y)=y-f(x)$ the following conditions are equivalent:

a) $\gamma(x)>f(x)$ for $x \neq 0$;

b) the field is outgoing along the graph of $f$ from the region $y \geq f(x)$ if $x<0$, and incoming if $x>0$. 
Proof. Put $\delta(x)=\gamma(x)-f(x)$. For some positive integer $n$ and some real $k \neq 0$ we have $\delta(x)=k x^{n}(1+\epsilon(x))$, where $\epsilon(x)$ tends to zero with $x$. Condition a) means that $n$ is even and $k>0$. Put

$$
D(x)=\frac{B(x, f(x))}{A(x, f(x))}-f^{\prime}(x) .
$$

Condition b) means that near the origin we have $D(x)>0$ for $x>0$ and $D(x)<0$ for $x<0$. A straightforward evaluation gives

$$
D(x)=n k x^{n-1}(1+\xi(x))
$$

where $\xi(x)$ tends to zero with $x$. Thus both conditions are equivalent.

An immediate application of this lemma and of its variants by space or time symmetries gives the following:

Proposition 2.6. Suppose $X_{1}^{0} a$ and $X_{2}^{0} a$ are tangent to $C_{0}{ }_{a}$ at the same point $P$ and that $X_{1}^{0} a(P), X_{2}^{0} a(P) \neq 0$. Let $y_{i}$ be the trajectory of $X_{i}^{0} a$ which passes trough $P$. Suppose that at $P, y_{1}, y_{2}$ have a contact of finite order $n$.Then, up to symmetries, if there is a canard trajectory for the field $Z^{0}$ a then one of the following conditions is satisfied:

a) $X_{1}^{0} a(P)=k X_{2}^{0} a(P)$ with $k>0$ and $y_{i} \subseteq D_{i}^{0} a$ for $i=1,2$ near $P$.

b) $X_{1}^{0} a(P)=k X_{2}^{0} a(P)$ with $k<0$ and $y_{i} \subseteq D_{1}^{0} a$ for $i=1,2$ near $P$.

Proof. a) If there is a canard trajectory and $X_{1}^{0} a(P)=k X_{2}^{0} a(P)$ with $k>0$ and $y_{1} \subseteq$ $D_{1}$ then $y_{2} \subseteq D_{2}$ because if it were not the case then the transition line in a neighborhood of $P$ should not have attractive or repulsive arcs. Similar arguments for $\mathrm{b}$ ).
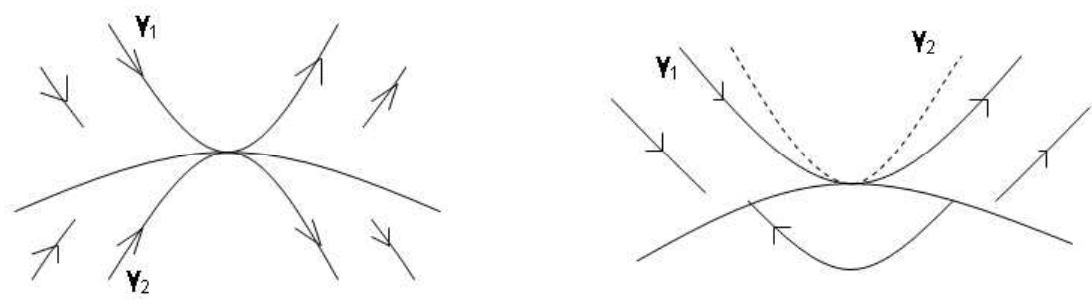

Figure 3. Proposition 2.6. On the left, the case a). On the right the case b), $y_{2}$ is represented by the dotted curve. In its upper part $D_{1}^{0} a$, on its lower part $D_{2}^{0} a$.

\subsection{Detailed study of an example with canards}

We consider a rather simple case where explicit computations illustrate clearly the canard phenomenon. Consider the fields

$$
X_{1}\left\{\begin{array} { l } 
{ \dot { x } = 1 } \\
{ \dot { y } = 2 x }
\end{array} \quad \text { and } \quad X _ { 2 } \left\{\begin{array}{l}
\dot{x}=1 \\
\dot{y}=-2 x
\end{array}\right.\right.
$$


and the family of transition curves defined by $h(x, y, a)=y+a x=0$, where $a$ is a real parameter. Then we have as collage of fields

$$
\left\{\begin{array}{l}
\dot{x}=1 \\
\dot{y}=2 x \varphi(\omega(y+a x))
\end{array}\right.
$$

and as the associated slow-fast system

$$
\left\{\begin{array}{l}
\dot{x}=1 \\
\dot{v}=\omega(2 x \varphi(v)+a)
\end{array}\right.
$$

If $a$ is not infinitesimal and near standard, then $C_{o_{a}}$ is attractive when $x<\frac{-\left.\right|^{0} a \mid}{2}$ and repulsive when $x>\frac{\left|{ }^{0} a\right|}{2}$, so no solution of $Z^{a}$ can follow two consecutive $\operatorname{arcs}$ of $C_{o_{a}}$ the first attractive the second repulsive and there are no canards. If $a=0$, call $A$ the region where $x<0$ and $B$ the region where $x>0$. Then the arc of the transition curve $C_{0}$ that lies in $A$ is attractive, and the one that lies in $B$ is repulsive, thus canards can occur. Indeed, the $x$-axis is the orbit of a canard trajectory of $Z^{0}$; moreover, by continuity there exist canard trajectories whenever $a \simeq 0$ is small enough. More precisely:

Theorem 2.7. $Z^{a}$ has canard trajectories if and only if either $a=0$ or there is a non infinitesimal positive number $M$ such that $|a|=e^{-M \omega}$.

Proof. Put $\varepsilon=\frac{1}{\omega}$; we use a Benoît-Callot microscope near the curve $v=0$ in the associated slow-fast system (2) that is the odd function $U=v^{[\varepsilon]}$ that for $v>0$ equals $\exp (\varepsilon \log (v))$. We obtain the system

$$
\left\{\begin{array}{l}
\dot{x}=1 \\
\dot{U}=|U|^{1-\omega}\left(2 x \varphi\left(U^{[\omega]}\right)+a\right)
\end{array}\right.
$$

Now $\varphi\left(U^{[\omega]}\right)=\varphi(0)+U^{[\omega]} \varphi^{\prime}\left(\theta U^{[\omega]}\right)$ with $0<\theta<1$. As $\varphi^{\prime}$ is standard and continuous, with $\varphi(0)=0$ and $\varphi^{\prime}(0)=1$, for $|U| \ll 1$ we get the the estimate $\varphi\left(U^{[\omega]}\right)=U^{[\omega]}(1+\phi)$ where the symbol $\phi$ stands for some unspecified infinitesimal. Hence, as long as $|U| \ll 1$ and $x$ is near standard the solutions of (3) and

$$
\left\{\begin{array}{l}
\dot{x}=1 \\
\dot{U}=U\left(2 x+\left(\frac{a^{[\varepsilon]}}{U}\right)^{[\omega]}\right)
\end{array}\right.
$$

are infinitely close. Thus for $|U| \ll a^{[\varepsilon]}, \dot{U}$ is infinitely large, while for $a^{[\varepsilon]} \ll|U| \ll 1$ we have $U(x) \simeq c \exp \left(x^{2}\right)$ where $c$ is a constant.

Due to the permanence principle, the approximation of any solution of (3) by some solution of (4) remains valid until $|U| \leq U_{0}$ with $U_{0}$ infinitely close to 1 . Now since $\varphi^{\prime}$ takes appreciable values on limited arguments, we deduce from

$$
\dot{U}=U\left(2 x \varphi^{\prime}\left(\theta U^{[\omega]}\right)+\left(\frac{a^{[\varepsilon]}}{U}\right)^{[\omega]}\right)
$$




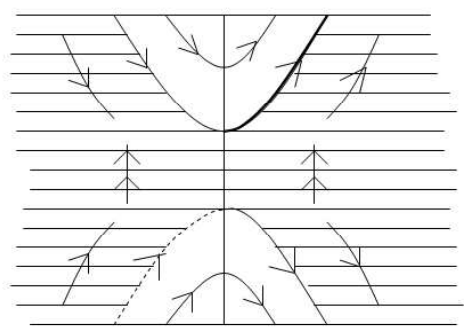

Figure 4. Shadows solutions of system (3).for $a>0$ and $a^{[\varepsilon]}<<1$.

that $\dot{U}$ is infinitesimal if and only if $x \simeq 0$. Thus the approximation remains true until $U_{0}=1$. In summary, for $a>0$ and $a^{[\varepsilon]} \ll 1$, the solutions of (3) are like those in FIG. 4, so there are canard trajectories.

For $a<0$ the picture is the same with reversed sense on vertical lines. If $|a|^{\varepsilon} \simeq 1$, there is no canard trajectory since for $x \gg 0, \dot{U}$ is not infinitesimal, so a trajectory from the attractive region cannot appreciably follow the repulsive one. Thus there is a canard trajectory if and only if $|a|^{\varepsilon} \ll 1$, or equivalently $|a|=\exp (-M \omega)$ with $M \gg 0$.

This theorem on the existence of canards gives a new point of view on discontinuous bifurcations; this claim will be detailed in Section 5 .

\section{Generic study of situations with canards}

Given $Z^{a}$ as in definition 2.1 and $a_{o}$ a standard value, assume the following necessary conditions for the existence of canard solutions of $Z^{a_{o}}$ around some standard point $\left(x_{o}, y_{o}\right)$ :

a) $h\left(x_{o}, y_{o}, a_{o}\right)=0, h_{y}\left(x_{o}, y_{o}, a_{o}\right) \neq 0$.

Ley $\bar{y}(x, a)$ denote the implicit function defined in a standard neighborhood of $\left(x_{o}, a_{o}\right)$ such that $h(x, \bar{y}(x, a), a)=0$ and $\bar{y}\left(x_{o}, a_{o}\right)=y_{o}$.

b) $X_{1}^{a_{o}}, X_{2}^{a_{o}}$ are tangent to the line $C_{a_{o}}$ at $\left(x_{o}, y_{o}\right)$.

c) $C_{a_{o}}$ is attractive for $x<x_{o}$ and repulsive for $x>x_{o}$.

This condition can be expressed using the functions

$$
\begin{aligned}
& F(x, a)=\left\langle\operatorname{grad} h(x, \bar{y}(x, a), a), X_{1}^{a}(x, \bar{y}(x, a))\right\rangle, \\
& G(x, a)=\left\langle\operatorname{grad} h(x, \bar{y}(x, a), a), X_{2}^{a}(x, \bar{y}(x, a))\right\rangle .
\end{aligned}
$$

Then we need $F\left(x, a_{o}\right)<0$ for $x<x_{o}$ and $F\left(x, a_{o}\right)>0$ for $x>x_{o}$, and the opposite signs for $G(x, a)$.

Set $m(x, v, a)=F(x, a)+G(x, a)+\varphi(v)[F(x, a)-G(x, a)]$ and

$$
v_{o}=\varphi^{-1}\left(-\frac{F_{x}\left(x_{o}, a_{o}\right)+G_{x}\left(x_{o}, a_{o}\right)}{F_{x}\left(x_{o}, a_{o}\right)-G_{x}\left(x_{o}, a_{o}\right)}\right)
$$

Under these conditions and with these notations we have 
Theorem 3.1. Suppose $F_{x}\left(x_{o}, a_{o}\right)>0, G_{x}\left(x_{o}, a_{o}\right)<0$ and $m_{a}\left(x_{o}, v_{o}, a_{o}\right) \neq 0$. Then in the three following cases there exists $a$ value $a \simeq a_{o}$ such that $Z^{a}$ admits canard solutions:

a) $f^{1}\left(x_{o}, y_{o}, a_{o}\right)>f^{2}\left(x_{o}, y_{o}, a_{o}\right)$ and

$$
\frac{f^{1}\left(x_{o}, y_{o}, a_{o}\right)+f^{2}\left(x_{o}, y_{o}, a_{o}\right)}{f^{1}\left(x_{o}, y_{o}, a_{o}\right)-f^{2}\left(x_{o}, y_{o}, a_{o}\right)}>\frac{F_{x}\left(x_{o}, a_{o}\right)+G_{x}\left(x_{o}, a_{o}\right)}{F_{x}\left(x_{o}, a_{o}\right)-G_{x}\left(x_{o}, a_{o}\right)}
$$

b) $f^{1}\left(x_{o}, y_{o}, a_{o}\right)<f^{2}\left(x_{o}, y_{o}, a_{o}\right)$ and

$$
\frac{f^{1}\left(x_{o}, y_{o}, a_{o}\right)+f^{2}\left(x_{o}, y_{o}, a_{o}\right)}{f^{1}\left(x_{o}, y_{o}, a_{o}\right)-f^{2}\left(x_{o}, y_{o}, a_{o}\right)}<\frac{F_{x}\left(x_{o}, a_{o}\right)+G_{x}\left(x_{o}, a_{o}\right)}{F_{x}\left(x_{o}, a_{o}\right)-G_{x}\left(x_{o}, a_{o}\right)}
$$

c) $f^{1}\left(x_{o}, y_{o}, a_{o}\right)=f^{2}\left(x_{o}, y_{o}, a_{o}\right)>0$

Proof. The associated slow-fast system of $Z^{a}$ is

$$
\left\{\begin{array}{l}
\dot{x}=A(x, v) \\
\varepsilon \dot{v}=h_{x}(x, \bar{Y}, a) A(x, v)+h_{y}(x, \bar{Y}, a) B(x, v)
\end{array}\right.
$$

with

$$
\begin{gathered}
\alpha(v)=\frac{1+\varphi(v)}{2}, \quad \beta(v)=\frac{1-\varphi(v)}{2}, \quad \varepsilon=\frac{1}{\omega} \\
A(x, v)=\alpha(v) f^{1}(x, \bar{Y}, a)+\beta(v) f^{2}(x, \bar{Y}, a) \\
B(x, v)=\alpha(v) g^{1}(x, \bar{Y}, a)+\beta(v) g^{2}(x, \bar{Y}, a)
\end{gathered}
$$

Once $h_{y}\left(x_{o}, y_{o}, a_{o}\right) \neq 0, \bar{Y}=\bar{Y}(x, z, a)$ is defined implicitly by $z=h(x, y, a)$ in a standard neighborhood of $\left(x_{o}, 0, a_{o}\right)$, making $z=\varepsilon v$ we have $\bar{Y}(x, \varepsilon v, a)$ defined implicitly by $\varepsilon v=h(x, y, a)$ and such that

$$
\bar{Y}(x, 0, a)=\bar{y}(x, a) .
$$

Using a Taylor expansion we obtain

$$
\left\{\begin{array}{l}
\dot{x}=\alpha(v) f^{1}(x, \bar{y}, a)+\beta(v) f^{2}(x, \bar{y}, a)+\varepsilon r(x, v, a) \\
\varepsilon \dot{v}=\frac{F(x, a)+G(x, a)+\varphi(v)[F(x, a)-G(x, a)]}{2}+\varepsilon \bar{r}(x, v, a)
\end{array}\right.
$$

where $r, \bar{r}$ are limited $C^{\infty}$ functions with limited derivatives. In order to complete the proof of the theorem we shall use the theorem of elementary fields given in Appendix A.

In the present context $n, p, q$ are chosen as follows

$$
\begin{gathered}
n(x, v, a)=\alpha(v) f^{1}(x, \bar{y}, a)+\beta(v) f^{2}(x, \bar{y}, a) \\
p(x, a)=F(x, a)+G(x, a), \quad q(x, a)=F(x, a)-G(x, a)
\end{gathered}
$$

The slow curve of the associated slow-fast system has no pole since $q\left(x_{o}, a_{o}\right)=0$ implies

$$
p\left(x_{o}, a_{o}\right)=0, \quad q_{x}\left(x_{o}, a_{o}\right)>0 \quad \text { and } \quad\left|\frac{p_{x}\left(x_{o}, a_{o}\right)}{q_{x}\left(x_{o}, a_{o}\right)}\right|<1 .
$$


On the other hand,

$$
\begin{aligned}
& p\left(x, a_{o}\right)=\left(x-x_{o}\right)\left[F_{x}\left(x_{o}, a_{o}\right)+G_{x}\left(x_{o}, a_{o}\right)+\xi(x)\right] \\
& q\left(x, a_{o}\right)=\left(x-x_{o}\right)\left[F_{x}\left(x_{o}, a_{o}\right)-G_{x}\left(x_{o}, a_{o}\right)+\phi(x)\right]
\end{aligned}
$$

with $\xi(x), \phi(x) \rightarrow 0$ when $x \rightarrow x_{o}$ and $\xi\left(x_{o}\right)=\phi\left(x_{o}\right)=0$. As a consequence, for $a=a_{o}$ the slow curve must be of the form $x=x_{o}$ or

$$
\varphi(v)=-\frac{F_{x}\left(x_{o}, a_{o}\right)+G_{x}\left(x_{o}, a_{o}\right)+\xi(x)}{F_{x}\left(x_{o}, a_{o}\right)-G_{x}\left(x_{o}, a_{o}\right)+\phi(x)},
$$

and $\left(x_{o}, v_{o}\right)$ is a Morse point as a consequence of $q_{x}\left(x_{o}, a_{o}\right)>0$. The first hypothesis of the elementary fields theorem will be verified if

$$
n\left(x, v,{ }^{o} a\right) q_{x}\left(x_{o},{ }^{o} a\right)>0
$$

which is the case in the three conditions stated in the theorem; the second hypothesis follows directly from the given hypothesis.

In the following we will study the existence of canards when the function $\varphi$ is not strictly increasing, and has $2 n+1$ zeros with $n$ standard. Of course the characteristic that when $h(x, y, a)>>0$ we have $Z^{a} \simeq X_{1}^{a}$ and when $h(x, y, a)<<0$ we have $Z^{a} \simeq X_{2}^{a}$ still remains. If

$$
m<-\frac{F_{x}\left(x_{o}, a_{o}\right)+G_{x}\left(x_{o}, a_{o}\right)}{F_{x}\left(x_{o}, a_{o}\right)-G_{x}\left(x_{o}, a_{o}\right)}<M
$$

with $M=\min \left\{\varphi\left(x_{i}\right)\right\}$ where $x_{i}$ is a local maximum of $\varphi$ and $m=\max \left\{\varphi\left(y_{i}\right)\right\}$ where $y_{i}$ is a local minimum of $\varphi$ then, under the same conditions of Theorem 3.1, the slow curve of the associated slow-fast system must be of the form $x=x_{o}$ and $2 n+1$ smooth curves that are transversal to $x=x_{o}$ in the points $\left(x_{o}, v_{i}\right)$ such that

$$
\varphi\left(v_{i}\right)=\frac{F_{x}\left(x_{o}, a_{o}\right)+G_{x}\left(x_{o}, a_{o}\right)}{G_{x}\left(x_{o}, a_{o}\right)-F_{x}\left(x_{o}, a_{o}\right)} .
$$

and applying the theorem of elementary fields locally to each of the $2 n+1$ smooth curves, they will be followed by canards or faux canards whether $\varphi^{\prime}\left(v_{i}\right)>0$ or $\varphi^{\prime}\left(v_{i}\right)<0$.

\section{Comparative study with sliding motion and equivalent control}

In this section we make a comparative study between slow dynamics obtained in collage of fields and sliding motion in Fillipov's theory. We also study Utkin's equivalent control smoothing the discontinuous control

Definition 4.1. Given the slow-fast system

$$
\left\{\begin{array}{c}
\dot{x}=f(x, y) \\
\epsilon \dot{y}=g(x, y)
\end{array}\right.
$$

with $f, g S^{1}$-functions, the curve $C$ defined by $g_{o}(x, y)=0$, where $g_{o}$ is the shadow of $g$ is named the slow curve of the slow-fast system. 
Definition 4.2. The slow dynamics of the system given in Definition 4.1 is the vector field tangent to $C$, whose image by means of the projection $(x, y) \rightarrow x$ leads to the differential equation $\dot{x}=f_{o}(x, \bar{y}(x))$ defined in the neighborhood of each $\left(x_{o}, y_{o}\right)$ where $\left(g_{o}\right)_{y}\left(x_{o}, y_{o}\right) \neq 0, \bar{y}(x)$ is defined by $g_{o}(x, y)=0$ and $f_{o}$ is the shadow of $f$.

An important characteristic of the slow dynamics is that, if $\gamma:] a, b\left[\rightarrow \mathbb{R}^{2}\right.$ is a solution of the slow-fast system such that $\gamma\left(t_{o}\right) \in$ hal $(C)$, where

$$
\text { hal }(C)=\left\{(x, y): g_{o}(x, y) \simeq 0\right\} \text {, }
$$

then for limited $t \geq t_{o} \gamma(t) \simeq \lambda(t)$ where $\lambda$ is the solution of the slow-fast dynamics with initial condition ${ }^{o} \gamma\left(t_{o}\right)=\lambda\left(t_{o}\right)$. For details see [3, 6].

If $a \simeq a_{o}$ and $a_{o}$ is a standard value then the slow dynamics of $Z^{a_{o}}$ and $Z^{a}$ are the same, therefore we study one slow dynamics say that of $Z^{a_{o}}$ and we omit the subscript $a_{o}$.

Definition 4.3. In variable structure systems, the sliding motion associated with $Z$ is the dynamics defined by the convex combination of $X_{1}, X_{2}$ which is tangent to the transition line $C$ when $C$ is attractive.

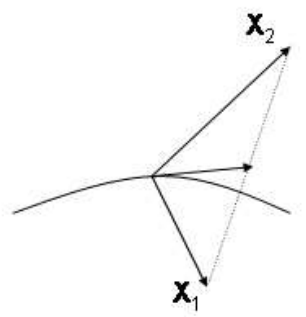

Figure 5. Sliding dynamics.

In the following we will use the same notations as in Section 3. We suppose that $h_{y}(x, y) \neq 0$ with $C=\{(x, y): h(x, y)=0\}$ and we let $\bar{y}(x)$ denote the function defined implicitly by $h(x, y)=0$. Then the sliding motion is given by

$$
\left\{\begin{array}{l}
\dot{x}=\alpha_{1}(x) f^{1}(x, \bar{y}(x))+\beta_{1}(x) f^{2}(x, \bar{y}(x)) \\
\dot{y}=\alpha_{1}(x) g^{1}(x, \bar{y}(x))+\beta_{1}(x) g^{2}(x, \bar{y}(x))
\end{array}\right.
$$

where

$$
\alpha_{1}(x)=\frac{-G(x)}{F(x)-G(x)}, \quad \text { and } \quad \beta_{1}(x)=\frac{F(x)}{F(x)-G(x)}
$$

In the following we will analyze the dynamics of the solutions of $Z$ in the halo of the slow curve of the associated slow-fast system of $Z$.

Theorem 4.4. If $\phi$ is a solution of $Z$ such that $\phi\left(t_{o}\right)$ belongs to the halo of the slow curve of the associated slow-fast system of $Z$, and $\psi(t)$ is the solution of the sliding motion associated with $Z$ such that ${ }^{\circ} \phi\left(t_{o}\right)=\psi\left(t_{o}\right)$ then for all $t \geq t_{o}, \phi(t) \simeq \psi(t)$ when $C$ is attractive. 
Proof. Using arguments similar to those in the proof of theorem 3.1 we obtain for the associated slow-fast system

$$
\left\{\begin{array}{l}
\dot{x}=\alpha(v) f^{1}(x, \bar{y}(x))+\beta(v) f^{2}(x, \bar{y}(x))+\varepsilon r(x, v) \\
\varepsilon \dot{v}=\frac{F(x)+G(x)+\varphi(v)[F(x)-G(x)]}{2}+\varepsilon \bar{r}(x, v)
\end{array}\right.
$$

where $r, \bar{r}$ are limited $C^{\infty}$ functions with limited derivatives. The slow curve is given by

$$
\varphi(v)=\frac{-F(x)-G(x)}{F(x)-G(x)}
$$

so we have as first projection of the slow motion

$$
\dot{x}=\alpha_{1}(x) f^{1}(x, \bar{y}(x))+\beta_{1}(x) f^{2}(x, \bar{y}(x)) .
$$

This is precisely the first projection of the sliding motion.

This theorem remains valid when the function $\varphi$ is not strictly increasing, and has $2 n+1$ zeros with $n$ standard.

The connection of the dynamics of $Z$ in the halo of the slow curve of the associated slow-fast system and the dynamics outside the

$$
\frac{1}{\omega} \operatorname{galaxy}(C)=\{(x, y): \omega h(x, y)=L\}
$$

where $L$ stands for a limited number, $Z \simeq X_{1}$ when $h(x, y)>0$ and $Z \simeq X_{2}$ when $h(x, y, a)<0$ is due to the fact that if $\phi$ is a solution of (8) such that $\phi\left(t_{o}\right)=\left(x_{o}, v_{o}\right)$ is near standard and does not belong to the halo of the slow curve of the system (8) then $x(t) \simeq x_{o}$; by permanence (Feherele's Principle) there exist two instants $t_{-}, t_{+} \simeq 0$ such that when $t \in\left[t_{-}, t_{+}\right], x(t) \simeq x_{o}, v\left(t_{-}\right)$is unlimited and $\left(x\left(t_{+}\right), v\left(t_{+}\right)\right)$belongs to the halo of the slow curve of (8). For more details see [3, 6].

One point must be stressed. As a matter of fact we have a class of collage of fields depending on which particular functions $\varphi$ and $h$ are chosen; examples of functions $\varphi$ are

$$
\text { th }(x), \quad \frac{x}{\sqrt{1+x^{2}}}
$$

and more generally given $\varphi$,

$$
\theta(x)=\varphi(x)\left(1-\varphi^{2}(x)+\varphi^{4}(x)\right)
$$

obeys the conditions required for the function $\varphi$. Obviously the process can be repeated. In spite of that Theorem 4.4 remains valid, same remarks can be done when $\varphi$ has $2 n+1$ zeros.

When the fields $X_{1}, X_{2}$ are not standard Theorem 4.4 is no longer valid as the following example shows: if

$$
X_{1}\left\{\begin{array}{l}
\dot{x}=\varphi(\omega y) \\
\dot{y}=-1
\end{array}, \quad X_{2}\left\{\begin{array}{l}
\dot{x}=\varphi(\omega y) \\
\dot{y}=k
\end{array}, \quad h(x, y)=y\right.\right.
$$

and $k>1$ is a standard constant, then there is no sliding motion and the slow motion of the associated slow-fast system is given by

$$
\dot{x}=\frac{k-1}{k+1} \text {. }
$$


In control theory there are situations where the control $u$ of the system changes very quickly in a curve $C$ given by $C=\{(x, y): h(x, y)=0\}$ the transition being usually modelled in a discontinuous way.

In [17] Utkin gives a formal procedure to assign a dynamics on $C$, the equivalent control method. More precisely, given the system

$$
\left\{\begin{array}{l}
\dot{x}=f(x, y, u) \\
\dot{y}=g(x, y, u)
\end{array}\right.
$$

where $u=u_{1}(x, y)$ for $h(x, y)>0$ and $u=u_{2}(x, y)$ for $h(x, y)<0$, the equivalent control is defined by

$$
h_{x}(x, \bar{y}(x)) f(x, \bar{y}(x), u)+h_{y}(x, \bar{y}(x)) g(x, \bar{y}(x), u)=0
$$

where $\bar{y}(x)$ is defined by $h(x, y)=0$. Roughly speaking, $u$ is a convex combination of $u_{1}, u_{2}$ such that the given system will be tangent to $C$ for each point on $C$, when $C$ is attractive.

In the following we smooth out the transition of the control $u$ and obtain the equivalent control dynamics as slow dynamics of a slow-fast system. We define

$$
u=\frac{1+\varphi(\omega h(x, y))}{2} u_{1}(x, y)+\frac{1-\varphi(\omega h(x, y))}{2} u_{2}(x, y)
$$

and by making $v=\omega h(x, y)$ we obtain the slow-fast system

$$
\left\{\begin{array}{l}
\dot{x}=f(x, \bar{y}(x), A(x, v))+\varepsilon r(x, v) \\
\dot{v}=w[B(x, v)+\varepsilon \bar{r}(x, v)]
\end{array}\right.
$$

where

$$
A(x, v)=\frac{1+\varphi(\omega h(x, \bar{y}(x)))}{2} u_{1}(x, \bar{y}(x))+\frac{1-\varphi(\omega h(x, \bar{y}(x)))}{2} u_{2}(x, \bar{y}(x))
$$

and

$$
B(x, v)=h_{x}(x, \bar{y}(x)) f(x, \bar{y}(x), A(x, v))+h_{y}(x, \bar{y}(x)) g(x, \bar{y}(x), A(x, v)) .
$$

The slow curve is precisely the definition of equivalent control and as a consequence slow dynamics is the equivalent control dynamics for each solution of $h_{x}(x, \bar{y}(x)) f(x, \bar{y}(x), u)+$ $h_{y}(x, \bar{y}(x)) g(x, \bar{y}(x), u)=0$ when all data are standard.

In this situation we can associate a collage of fields

$$
X_{i=1,2}\left\{\begin{array}{l}
\dot{x}=f\left(x, y, u_{i=1,2}\right) \\
\dot{y}=g\left(x, y, u_{i=1,2}\right)
\end{array}\right.
$$

through the transition line $C$. Slow dynamics of the associated slow-fast system and equivalent control dynamics are usually different. It is straightforward to see that, when $f, g$ are linear in $u$, then there is coincidence between equivalent control dynamics and the slow dynamics of the associated slow-fast system.

In the following example equivalent control dynamics is not uniquely determined. Consider the system.

$$
\left\{\begin{array}{l}
\dot{x}=1+u \\
\dot{y}=-\sin (a u)
\end{array}\right.
$$


with $a>2 \pi, \sin (a)>0$ and $u=1$ if $y>0$ and $u=-1$ if $y<0$. Each $u=\frac{n \pi}{a}$ with $n \pi<a$ and $n$ an integer, gives origin to a different equivalent control dynamics. These dynamics appear in the

$$
\frac{1}{\omega} \operatorname{galaxy}(y=0)=\{(x, y): \omega y=L\}
$$

where $L$ stands for a limited number, of the system

$$
\left\{\begin{array}{l}
\dot{x}=1+\varphi(v) \\
\dot{v}=-\omega \sin (a \varphi(v))
\end{array}\right.
$$

that is obtained letting $u=\varphi(\omega y)$ and $v=\omega y$. The fact that equivalent control dynamics appears in the $\frac{1}{\omega}$ galaxy $(C)$ justifies à posteriori equivalent control method, once all equivalent control dynamics appears in a narrow band around the separation line $C$.

\section{Canards and discontinuous bifurcations}

In this section we will return to the example given in Section 2.3 to show how the existence of canards can be used to smooth discontinuous bifurcations in plane variable structure systems.

In [12], $a_{o}$ is defined as a bifurcation value of $Z^{a}$ if by an arbitrarily small perturbation of $a_{o}$ a topologically non-equivalent system is obtained.

This is the case for $a=0$ in the system (1) since in that case the whole transition line is a sliding segment which is not the case for $a \neq 0$ no matter how small $a$ is. As a consequence, in the discontinuous approach for $a \neq 0$ an orbit coming from the attractive region cannot follow the repulsive one.

Theorem 2.7 explains the transition in a smooth way between the cases where $a>$ $0, a \simeq 0$ and $a<0, a \simeq 0$ both cases with no canard solutions, indeed for $a>0, a^{\varepsilon} \ll 1$ there exists a funnel with neck at $x \simeq \sqrt{-\varepsilon \log (a)}$ and an anti-funnel with neck at $x \simeq-\sqrt{-\varepsilon \log (a)}$ which means that all the trajectories of the region filled with lines in FIG. 4 with $x<0$ leave the band $|U| \leq 1$ along the heavy curve and all the trajectories of the region filled with lines with $x>0$ came from the halo of the dotted curve.

In the system (1) this means that all the trajectories of the region filled with lines in FIG. 6, left, with $x<0$ leave the halo of the transition line along the heavy curve and all the trajectories of the region filled with lines with $x>0$ came from the halo of the dotted curve.

For $a<0,|a|^{\varepsilon} \ll 1$ we have a similar situation described in FIG. 6, right.

As $a$ approaches zero the vector field admits longer and longer canard solutions that can be viewed as the missing link in discontinuous bifurcations.

In [12] a point $P$ is defined as a pseudo-equilibrium if it is a point of the separation line where the vectors $X_{1}(P), X_{2}(P)$ are transversal to the separation line and anticollinear, when both vectors $X_{1}(P), X_{2}(P)$ are tangent to the separation line, $P$ is called a singular sliding point. 

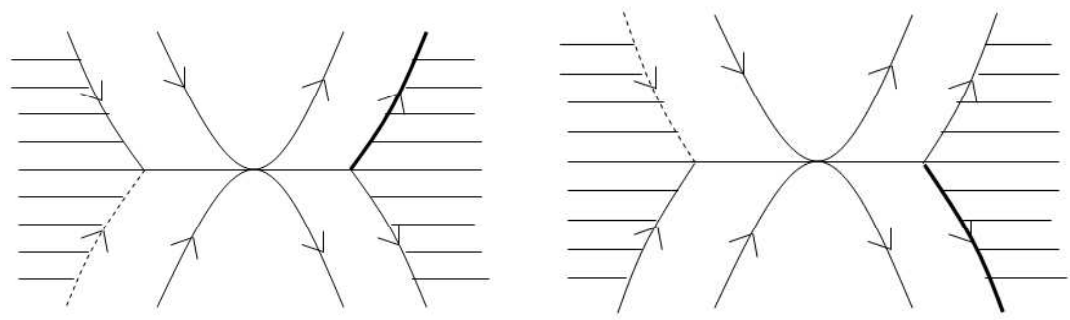

Figure 6. Funnel and anti-funnel: On the left the case $a>0$, on the right the case $a<0$.

From the discontinuous point of view, the system

$$
X_{1}\left\{\begin{array}{l}
\dot{x}=1 \\
\dot{y}=a-x
\end{array} \quad, \quad X_{2}\left\{\begin{array}{l}
\dot{x}=-1 \\
\dot{y}=a-x
\end{array} \quad, \quad h(x, y)=y-\frac{x^{3}}{3}+x\right.\right.
$$

exhibits a catastrophic disappearance of a repulsive pseudo-equilibrium when $a$ approaches $1(a<1)$. This is due to the fact that the transition between repulsive pseudo-equilibrium points for $a<1$ and attractive pseudo-equilibrium points for $a>1$ is done through a singular sliding point. In collage of fields the transition is done through a Hopf -Andronov bifurcation.

\section{Concluding remarks}

Continuous approximations of variable structure systems are rarely used. The main objections against their use are presented in [12], "continuous approximations give rise to stiff differential equations and worse than that, the most interesting sliding bifurcation phenomena are absent". Continuous approximations of variable structure systems through the use of nonstandard analysis avoid problems associated with stiff differential equations as it was seen in section 4 and, when a canard phenomenon arises, it smooths discontinuous bifurcations. An alternative approach to get continuous approximations of variable structure systems is given by the regularization method, introduced by Sotomayor and Teixeira $[5,16]$

\section{A. The theorem of Elementary Fields}

This is a result of M. Diener [7] that is a basic tool for the study of the existence of canard solutions in rather general situations. It gives sufficient existence conditions when the slow-fast vector field has the following geometric behavior as described in FIG. 7 for some standard value of the parameter.

We give here a slightly more general version of the theorem, which can be proved along the same lines as the original version. 

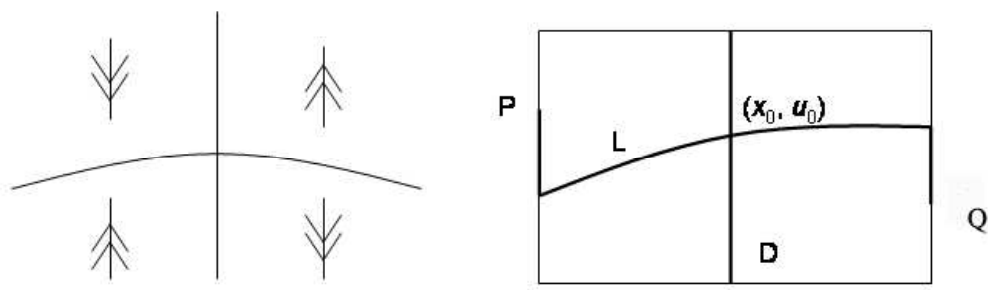

Figure 7. On the left an elementary field. On the right, a canard solution.

Let us call elementary a slow-fast vector field $V_{a}$ depending on a parameter $a$, which has an associated system of the following type

$$
\left\{\begin{aligned}
\dot{x} & =n(x, v, a)+\varepsilon r(x, v, a) \\
\varepsilon \dot{v} & =m(x, v, a)+\varepsilon \bar{r}(x, v, a)
\end{aligned}\right.
$$

with $m(x, v, a)=p(x, a)+\varphi(v) q(x, a)$, where $n, p, q$ are standard $C^{\infty}$ functions $r$ and $\bar{r}$ are $C^{\infty}$ functions taking limited values and limited first derivatives. The slow curve for a near standard value of the parameter $a$ will be given by

$$
p\left(x,{ }^{o} a\right)+\varphi(v) q\left(x,{ }^{o} a\right)=0 .
$$

We suppose that the slow curve has no pole on some standard interval $I=\left[x_{-}, x_{+}\right]$, with $x_{-} \neq x_{+}$, in the following sense: if $q\left(x_{1},{ }^{o} a\right)=0$ for some $x_{1} \in I$ then

$$
p\left(x_{1},{ }^{o} a\right)=0, \quad q_{x}\left(x_{1},{ }^{o} a\right) \neq 0 \quad \text { and } \quad\left|\frac{p_{x}\left(x_{1}, o a\right)}{q_{x}\left(x_{1},{ }^{o} a\right)}\right|<1 .
$$

Thus the slow curve is either a smooth curve $L$ transverse to the verticals over the interval $I$, or is the union of a smooth curve $L$ and a vertical line $D$ for each root $x_{1}$ of $q\left(.{ }^{o} a\right)=$ 0 . Call $R=I \times\left[U_{-}, U+\right]$ a standard rectangle such that, for $a={ }^{o} a$, the interior of $R$ contains a unique Morse point $\left(x_{1}, v_{1}\right)$ of the slow curve, and no other singularity, for which the component $L$ meets each vertical edge of the boundary of $R$ at an inner point of this edge. A Morse point of the slow curve is a point $\left(x_{1}, v_{1},{ }^{o} a\right)$ where $m_{x}=0$ and $m_{x x} m_{v v}-m_{x v}^{2}<0$.

Under these hypotheses we have

Theorem A.1. Suppose that $n\left(x, v,{ }^{o} a\right) q_{x}\left(x_{1},{ }^{o} a\right)>0$ for $(x, v) \in R$ and $m_{a}\left(x_{1}, v_{1},{ }^{o} a\right) \neq$ 0. Then

a) There exists a standard neighborhood $W$ of ${ }^{\circ}$ a such that each $a \in W$ for which there exists a canard solution is infinitely near ${ }^{\circ} a$.

b) For each point $P$ on the left vertical edge of $R$ and each point $Q$ on the right one, there exists a value a infinitely near ${ }^{\circ} a$ such that the field $V_{a}$ admits a canard solution that joins $P$ to $Q$ (see FIG. 7, right).

Acknowledgements. The author thanks Robert Lutz and Tewfik Sari for their constant support and encouragement. 


\section{B. References}

[1] L. G. Albuquerque, Collage des champs et phénomène canard, Thèse Mulhouse,1994

[2] A. A. Andronov, A. A. Vitt and S. E. Kaikin, Theory of Oscilators, Dover, New York, 1987.

[3] E. Benoit, Systèmes Lent-Rapides dans $\mathbb{R}^{3}$ et leurs canards, in III Rencontre de Géométie du Schnepfenried, Astérisque, volume 2, Société Mathématique de France,1982, pp. 159-191

[4] E. Benoît, J. L. Callot, F. Diener and M. Diener, Chasse aux canards, Collectanea Matematicae 32 (1981) 37-119.

[5] C. A. Buzzi, P. R. da Silva, M. A. Teixeira, A singular approach to discontinuous vector fi elds on the plane, J. Differential Equations, 231 (2006) 633-655.

[6] F. Diener and M. Diener (Eds.), Nonstandard Analysis in Practice, Universitext, SpringerVerlag, 1995.

[7] M. Diener, Etude Generique des Canards, Thèse, Irma,1981.

[8] M. Diener, The canard Unchained or How Fast/Slow Dynamical Systems Bifurcate, The Mathematical Intelligencer, Vol. 6,No 3 (1984) 38-49.

[9] A. F. Filippov, Differential Equations with Discontinuous Right-Hand Sides, Kluwer Academic, Dordrecht, 1988.

[10] I. Flugge-Lotz, Discontinuous Automatic Control, Princeton University Press, 1953.

[11] R. M. Goodwin, The non-linear accelerator and the peristence of business cycles, Econ. 19 (1951) 1-17.

[12] Yu. A. Kuznetsov, S. Rinaldi and A. Gragnani, One-parameter Bifurcations in Planar Filippov Systems, Int. J. Bifurcation \& Chaos 13 (2003) 2157-2188.

[13] R. Lutz, L. G. Albuquerque, Modern Infi nitesimals As a Tool To Match Intuitive And Formal reasoning In Analysis, Synthese 134 (2003) 325-351

[14] R. Lutz and M. Goze, Nonstandard Analysis: A Practical Guide with Applications, Lecture Notes in Mathematics, Vol. 881, Springer-Verlag, Berlin/New York, 1981.

[15] E. Nelson, Internal Set Theory, Bull. Amer. Math. Soc. 83. (1977) 1165-1198.

[16] J. Sotomayor, M. A. Teixeira, Regularization of discontinuous vector fi elds, in: International Conference on Differential Equations, Lisboa, 1996, pp 207-223.

[17] V. Utkin, Sliding Modes in Control and Optimization, Springer-Verlag, Berlin, 1992.

[18] F. Takens, Constrained equations: a study of certain implicit differential equations and their discontinuous solutions in: Structural stability, the theory of catastrophes and the applications in sciences. Springer lecture notes in math. 525 (1976) 143-234.

[19] F. Takens Implicit differential equations : some open problems in : Singularités d'applications différentiables, Plan sur Bex (1975). Springer lecture notes in math. 535 (1976) 237-253.

[20] A. K. Zvonkin and M. A. Shubin, Non-standard analysis and singular perturbations of ordinary differential equations, Russian Math. Surv. 39:2 (1984) 69-131. 